\title{
ARE WE READY TO INDICATE MYELODYSPLASTIC SYNDROME PATIENTS TO HEMATOPOIETIC STEM CELL TRANSPLANTATION BASED ON THEIR GENETIC STATUS?
}

\section{SHORT COMMUNICATION}

\author{
Mair Pedro de Souza', Fernanda Leite Souza Franceschi', Fernando Barroso Duarte ${ }^{2}$, Diego F. \\ Coutinho ${ }^{3}$, Beatrice Araujo Duarte ${ }^{4}$, Romelia Pinheiro Gonçalves ${ }^{5}$ e Vergilio Colturato $^{1}$
}

\begin{abstract}
1 Serviço de Transplante de Medula Óssea do Hospital Amaral Carvalho - Jaú, São Paulo - 2 Hospital Universitário Walter Cantídio/UFC, Departamento de Cirurgia - Fortaleza, Ceará - 3 Pediatric Translational Medicine Program, Memorial Sloan Kettering Cancer Center - New York, NY - 4 Aluna de graduação da Universidade Unichristus - Fortaleza, Ceará - 5 Universidade de Fortaleza/UFC, Departamento de Farmácia - Fortaleza, Ceará
\end{abstract}

\section{Correspondence to: mairepepa@gmail.com}

Myelodysplastic Syndrome (MDS) is a heterogeneous set of malignant disorders related to the impairment of the bone marrow (BM) and its functions. The occurrence of signs of ineffective hematopoiesis, with progressive BM failure; molecular and cytogenetic damages, and the risk of progression into Acute Myeloid Leukemia are meaningful hallmarks of the disease. MDS might occur with a wide spectrum of clinical presentations which may require different proposals as therapeutic approaches.

Nowadays, there are several systems of clinical stratification and prognostic prediction for MDS being the International Prognostic Scoring System (IPSS)[1], Revised-IPSS (IPSS-R), WHO-Classification-based Prognostic Scoring Systems, and the Global MD Anderson System (MDAPPS) widely used worldwide[2,3,4]. The evaluation by such systems frequently demands cytomorphological data, cytogenetic status, severity of cytopenias, age, transfusion requirement, and others. Although IPSS and IPSS-R are systems often used to stratify MDS patients, they have significant limitations given that those are only applied to newly diagnosed cases and overlook secondary MDS and patients under or after previous treatment.[5]

The use of current laboratory tools and efforts to implement and enrich information records of MDS patients improves the way how those data might be used to support clinically patients and allows us to understand better their relationship. A limited repertoire of genetic mutations is often associated specifically with MDS subsets, outcome, drug response, and clonal hematopoiesis age-related, which seems to be a pre-leukemic stage leading to MDS or other myeloid malignancies. In another hand, previous studies point out the possibility of using genetic information as well as novel algorithms to conduct therapeutic strategies. [6 7]

New generation sequencing (NGS) techniques have been widely used to genomic assessment of MDS patients and are being gradually incorporated in the Brazilian center. Its use impacts on diagnosis and also helps to discriminate between MDS and other myelopathies, such as aplastic anemia, idiopathic cytopenias, and myeloproliferative neoplasms. The knowledge of the genomic profile underlying MDS patients might improve the disease classification and identify target genes to drive specific therapies (IDH1/2 inhibitors, for example), as well as to assist the prognostic and track minimum residual disease, even though this is being still explored pre-clinically. [8 9]

Based on the discussion of the following case, a 69-year-old white man with no comorbid presented in May of 2018 mild neutropenia and drop-in hemoglobin levels, although no anemia observed. Complete Blood Count of the diagnosis sample showed Hemoglobin $=13.6 \mathrm{~g} / \mathrm{dl}$; Leukocytes = 3,200 / mm3; Neutrophils $=945 / \mathrm{mm} 3$; Monocytes $=750 / \mathrm{mm} 3$; Platelets $=168,000 / \mathrm{mm} 3$. Subsequent exams showed the maintenance of isolated neutropenia and monocytosis (745 to $2437 / \mathrm{mm} 3$, respectively). Peripheral Blood Immunophenotyping, Myelogram and Bone Marrow Biopsy led to the diagnosis of MDS/MPN, Refractory Cytopenia / Chronic Myelomonocytic Leukemia(CMML 0-OMS 2016). karyotype Normal; In situ hybridization panel for (5q31.2 (EGR1), 7q22 (RELN), 11q23.3 (MLL), 16p13.1 (MYH11), 16q22 (CBFB), 17p13.1 (TP53), 20q12 (PT- 
PRT) e20q13.1 (MYBL2) and RUNX1T1 / RUNX1 and PML / RARA rearrangements by fluorescence were negative. NGS evaluation showed the presence of a somatic variant in ZRSR2 gene (c.312+1G>T) with allele frequency (VAF) of $90.81 \%$. Also, another variant was observed in the gene RUNX1. Initially, the variant RUNX1 p.Leu56Ser was described as a somatic alteration identified in patients with AML and MDS (REf .: doi: 10.1038 / leu.2011.19; doi: 10.18632 / oncotarget.9026). Later, Drazer et. al. demonstrated for the first time the germline origin of this change (Ref $\therefore 10.1182$ / blood advances.2017013037), endorsed by a VAF of $40-60 \%$, commonly observed. However, according to the genomic databases, the population frequency of the p.Leu56Ser confirms this is a common polymorphism in southern Asia, Europe, and Latin America [Ref .: gnomAD; https://gnomad. broadinstitute.org]. In general, these data demonstrate the importance of curating properly the data obtained from NGS following rigorous criteria, especially when it pursues somatic variants.

The gene ZRSR2 plays an important role in spliceosome machinery, being then a critical partner over the RNA editing. 16171810 and shows frequently mutated in MDS affecting from 3 to $11 \%$ of cases. $[19,6]$ The variant c.312+1G>T observed in the patient herein discussed occurs in exon/intron boundary and has already been described in a patient with monoclonal gammopathy of undetermined significance (MGUS), an entity underlying to B cell malignancies (Ref .: doi: 10.1016 / j.stem.2017.07.010). Its high VAF might be explained once the ZRSR2 gene is within the chromosome $X$ (homozygous variant) and, possibly, it led to the clonal expansion of the malignant cell.

Herein, we had a 69-year-old patient, fully asymptomatic, ECOG 0, with no transfusion requirement, with history of neutropenia and monocytosis, cytomorphology and bone marrow immunophenotyping confirming MDS, normal karyotype, and without chromosome aberrations detected by FISH. The NGS identified the somatic mutation in ZRSR2.

When the therapeutic discussion involves the indication of HSCT, proposed with curative intentions, the decision-making process necessarily passes through another complex chain of information. We must consider aspects related to the disease,[2,21,23] patient, 16 and also to the availability of an appropriate donor (related, young, male)

If we use the criteria of the IPSS-R, adjusted for age, this is a low-risk patient. However, the gene alter- ation detected by NGS may indicate higher chance of leukemic transformation and poor overall survival. Disease Risk Index (DRI) indicators 2425 shows an intermediate risk condition for transplantation. If we evaluate the patient's condition and possible risk indicators using the "hematopoietic-cell-transplantation-specific-comorbidity-index" (HCT-I) $[26,16]$ the patient is classified as low risk.

With a curative potential, HSCT is usually indicated for patients under 75 years of age, most often recommended as first-line therapy (with or without previous treatments) in high-risk patients. In lower-risk patients, the usual practice is the indication of monitoring, with transplantation when there are signs of progression.27 Transplants, at the time of diagnosis, using reduced-intensity conditioning in patients over 60 years of age do not result in benefits for low or intermediate-risk groups.[28] A similar study showed that delaying transplantation was beneficial for low- and very low-risk patients, but not for intermediate-risk patients [29]

In the case under discussion, the finding of the mutation in ZRSR2 triggered the discussion about the possibility of transplant indication, due to concerns about the prospect of leukemic transformation. Adding the fact that the patient was in good performance for the procedure.

The lack of a suitable donor, and the real situation of a single haploidentical donor, must be included in the difficulties in decision making. A panel of experts in 2017 recommends that alternative donors be recommended only for high-risk patients.[30] The results with haploidentical transplants, in general, have shown improvements. They must be understood as an alternative, in the absence of a compatible donor.[31, 32]

The case leads us to reflect on the meaning of laboratory findings and their relevance when making therapeutic recommendations. Understanding the exact role of each finding in molecular genetics and its incorporation into the current risk stratification systems is an ongoing action that may result in an adequate therapeutic recommendation and in survival gain of MDS patients.[33] Great possibilities of using electronic tools and artificial intelligence algorithms are being proposed in the context of precision medicine.[34,35,36,37,] Seeking more consistent results involves recommending transplantation to well-selected patients, at the appropriate time, and promoting approaches in the pre- and post-transplant periods that can decrease recurrence rates. For 
example, identifying patients and situations which there may be benefits from isolated or combined strategies, involving infusion of donor lymphocytes and hypomethylating drugs.[30]

Consider the perspective that the molecular "target" drugs under development, new hypomethylating agents and formulations can bring benefits and promote changes in strategies.[38] Contextualizing HSCT in the complex information network, despite any exquisite assistance, should still be a medical decision wary to each patient.

The decision, shared with the patient, was not to perform the transplant and monitor him. After 24 months of follow-up after the diagnostic definition, he remains well, asymptomatic and without cytopenias.

\section{REFERENCES}

1. Greenberg, P. et al. International scoring system for evaluating prognosis in myelodysplastic syndromes. Blood (1997) doi: 10.1182 / blood. v89.6.2079.

2. Greenberg, PL et al. Revised international prognostic scoring system for myelodysplastic syndromes. Blood (2012) doi: 10.1182 / blood-2012-03-420489.

3. Della Porta, MG et al. Validation of WHO classification-based Prognostic Scoring System (WPSS) for myelodysplastic syndromes and comparison with the revised International Prognostic Scoring System (IPSS-R). A study of the International Working Group for Prognosis in Myelodyspla. Leukemia (2015) doi: 10.1038 / leu.2015.55.

4. Zeidan, AM et al. Comparison of risk stratification tools in predicting outcomes of patients with higher-risk myelodysplastic syndromes treated with azanucleosides. Leukemia (2016) doi: 10.1038 / leu.2015.283.

5. Nazha, A. et al. The efficacy of current prognostic models in predicting outcome of patients with myelodysplastic syndromes at the time of hypomethylating agent failure. Haematologica (2016) doi: 10.3324 / haematol.2015.140962.

6. Ogawa, S. Genetics of MDS. Blood (2019) doi: 10.1182 / blood-2018-10-844621.

7. Drusbosky, L. et al. iCare 1: A prospective clinical trial to predict treatment response based on genomics-informed computational biology in pa- tients with acute myeloid leukemia (AML) and myelodysplastic syndromes (MDS). J. Clin. Oncol. (2018) doi: 10.1200 / jco.2018.36.15_suppl.7024.

8. Lew, JL, Fenderson, JL \& Carmichael, MG Next-Generation Gene Sequencing Differentiates Hypoplastic Myelodysplastic Syndrome from Aplastic Anemia. Hawaii. J. Med. Public Health (2017).

9. Tobiasson, M. \& Kittang, AO Treatment of myelodysplastic syndrome in the era of next-generation sequencing. Journal of Internal Medicine (2019) doi: 10.1111 / joim.12893.

10.Ganguly, BB \& Kadam, NN Mutations of myelodysplastic syndromes (MDS): An update. $\mathrm{Mu}$ tation Research - Reviews in Mutation Research (2016) doi: 10.1016 / j.mrrev.2016.04.009.

11.Bejar, R. et al. Clinical effect of point mutations in myelodysplastic syndromes. N. Engl. J. Med. (2011) doi: 10.1056 / NEJMoa1013343.

12.Bejar, R. et al. Validation of a prognostic model and the impact of mutations in patients with lower-risk myelodysplastic syndromes. J. Clin. Oncol. (2012) doi: 10.1200 / JCO.2011.40.7379.

13.Della Porta, MG et al. Clinical effects of driver somatic mutations on the outcomes of patients with myelodysplastic syndromes treated with allogeneic hematopoietic stem-cell transplantation. J. Clin. Oncol. (2016) doi: 10.1200 / JCO.2016.67.3616.

14.Tefferi, A. et al. Mayo Alliance Prognostic Model for Myelodysplastic Syndromes: Integration of Genetic and Clinical Information. Mayo Clin. Proc. (2018) doi: 10.1016/ j.mayocp.2018.04.013.

15.Tsai, SC et al. Biological activities of RUNX1 mutants predict secondary acute leukemia transformation from chronic myelomonocytic leukemia and myelodysplastic syndromes. Clin. Cancer Res. (2015) doi: 10.1158 / 1078-0432.CCR14-2203.

16.Sorror, ML et al. Comorbidity-age index: A clinical measure of biologic age before allogeneic hematopoietic cell transplantation. J. Clin. Oncol. (2014) doi: 10.1200 / JCO.2013.53.8157.

17.Madan, V. et al. ZRSR2 Mutations Cause Dysregulated RNA Splicing in MDS. Blood (2014) doi: 10.1182 / blood.v124.21.4609.4609.

18. Hershberger, CE et al. The Biological and Clinical 
Implications of the Alternative Splicing Landscape of 1,258 Myeloid Neoplasm Cases. Blood (2019) doi: 10.1182 / blood-2019-128278.

19.Yoshida, K. et al. Frequent pathway mutations of splicing machinery in myelodysplasia. Nature (2011) doi: 10.1038 / nature10496.

20.Thol, F. et al. Frequency and prognostic impact of mutations in SRSF2, U2AF1, and ZRSR2 in patients with myelodysplastic syndromes. Blood (2012) doi: 10.1182 / blood-2011-12-399337.

21.Bejar, R. Clinical and genetic predictors of prognosis in myelodysplastic syndromes. Haematologica (2014) doi: 10.3324 / haematol.2013.085217.

22.Jaiswal, S. et al. Age-related clonal hematopoiesis associated with adverse outcomes. N. Engl. J. Med. (2014) doi: 10.1056 / NEJMoa1408617.

23.Steensma, DP Myelodysplastic syndromes current treatment algorithm 2018. Blood Cancer J. (2018) doi: 10.1038 / s41408-018-0085-4.

24.Armand, P. et al. A disease risk index for patients undergoing allogeneic stem cell transplantation. Blood (2012) doi: 10.1182 / blood-2012-03-418202.

25.Armand, P. et al. Validation and refinement of the Disease Risk Index for allogeneic stem cell transplantation. Blood (2014) doi: 10.1182 / blood-2014-01-552984.

26.Sorror, ML et al. Hematopoietic cell transplantation (HCT) -specific comorbidity index: A new tool for risk assessment before allogeneic HCT. Blood (2005) doi: 10.1182 / blood-2005-05-2004.

27.Cutler, CS et al. A decision analysis of allogeneic bone marrow transplantation for the myelodysplastic syndromes: Delayed transplantation for low-risk myelodysplasia is associated with improved outcome. Blood (2004) doi: 10.1182 / blood-2004-01-0338.

28.Koreth, J. et al. Role of reduced-intensity conditioning allogeneic hematopoietic stem-cell transplantation in older patients with de novo myelodysplastic syndromes: An international collaborative decision analysis. J. Clin. Oncol. (2013) doi: 10.1200 / JCO.2012.46.8652.

29.della Porta, MG et al. Decision analysis of allo- geneic hematopoietic stem cell transplantation for patients with myelodysplastic syndrome stratified according to the revised International Prognostic Scoring System. Leukemia (2017) doi: 10.1038 / leu. 2017.88.

30.De Witte, T. et al. Allogeneic hematopoietic stem cell transplantation for MDS and CMML: Recommendations from an international expert panel. Blood (2017) doi: 10.1182 / blood-2016-06-724500.

31.Ciurea, sO et al. Haploidentical transplant with posttransplant cyclophosphamide vs matched unrelated donor transplant for acute myeloid leukemia. Blood (2015) doi: 10.1182 / blood-2015-04-639831.

32.Wang, Y. et al.. Haploidentical transplant for myelodysplastic syndrome: Registry-based comparison with identical sibling transplant. Leukemia (2016) doi: 10.1038 / leu.2016.110.

33.Du, M. yi et al. Evaluation of different scoring systems and gene mutations for the prognosis of myelodysplastic syndrome (MDS) in Chinese population. J. Cancer (2020) doi: 10.7150 / jca.30363.

34.Stein, AS et al. Superior therapy response predictions for patients with myelodysplastic syndrome (MDS) using Cellworks Singula: MyCare-009-02. J. Clin. Oncol. (2020) doi: 10.1200 / jco.2020.38.15_suppl.e19528.

35.Cargo, C. \& Bowen, D. Individual risk assessment in MDS in the era of genomic medicine. Seminars in Hematology (2017) doi: 10.1053 / j.seminhematol.2017.07.002.

36.Nazha, A. \& Sekeres, MA Precision Medicine in Myelodysplastic Syndromes and Leukemias: Lessons from Sequential Mutations. Annual Review of Medicine (2017) doi: 10.1146 / annurev-med-062915-095637.

37.Parisi, S. et al. Current therapy and new drugs: A road to personalized treatment of myelodysplastic syndromes. Expert Review of Precision Medicine and Drug Development (2018) doi: 10.1080 / 23808993.2018 .1419820$.

38.Hellström-Lindberg, E., Tobiasson, M. \& Greenberg, P. Myelodysplastic syndromes: Moving towards personalized management. Haematologica (2020) doi: 10.3324 / haematol.2020.248955. 